\title{
Comparison of factor-analytic and reduced rank models for test-day milk yield in Gyr dairy cattle (Bos indicus)
}

\author{
R.J. Pereira', D.R. Ayres ${ }^{1}$, L. El Faro ${ }^{2}$, R.S. Verneque ${ }^{3}$, A.E. Vercesi Filho ${ }^{2}$ \\ and L.G. Albuquerque ${ }^{1}$ \\ ${ }^{1}$ Faculdade de Ciências Agrárias e Veterinárias, \\ Universidade Estadual Paulista Júlio de Mesquita Filho, Jaboticabal, SP, Brasil \\ ${ }^{2}$ Agência Paulista de Tecnologia dos Agronegócios, Ribeirão Preto, SP, Brasil \\ ${ }^{3}$ Embrapa Gado de Leite, Juiz de Fora, MG, Brasil \\ Corresponding author: R.J. Pereira \\ E-mail: rodjunper@gmail.com
}

Genet. Mol. Res. 12 (3): 4036-4045 (2013)

Received March 2, 2013

Accepted July 29, 2013

Published September 27, 2013

DOI http://dx.doi.org/10.4238/2013.September.27.5

\begin{abstract}
We analyzed 46,161 monthly test-day records of milk production from 7453 first lactations of crossbred dairy Gyr (Bos indicus) $\mathrm{x}$ Holstein cows. The following seven models were compared: standard multivariate model (M10), three reduced rank models fitting the first 2,3 , or 4 genetic principal components, and three models considering a 2-, 3-, or 4-factor structure for the genetic covariance matrix. Full rank residual covariance matrices were considered for all models. The model fitting the first two principal components (PC2) was the best according to the model selection criteria. Similar phenotypic, genetic, and residual variances were obtained with models M10 and PC2. The heritability estimates ranged from 0.14 to 0.21 and from 0.13 to 0.21 for models M10 and PC2, respectively. The genetic correlations obtained with model PC2 were slightly higher than those estimated with model M10. PC2 markedly reduced the number of parameters estimated and the time spent to reach convergence. We concluded that two principal
\end{abstract}


components are sufficient to model the structure of genetic covariances between test-day milk yields.

Key words: Factor analysis; Principal components; Milk production

\section{INTRODUCTION}

In tropical regions, milk production systems are based on pastures as the main food source for animals. In these systems, the genetic material basically comprises dairy Zebu cattle and their crossbreds. In Brazil, a National Breeding Program (PNMGL) exists for Gyr dairy cattle (Bos indicus), whose main objective is the genetic improvement of the breed through the identification and selection of sires that are genetically superior in terms of production, conformation and management traits (Verneque et al., 2011). Among the 23 traits evaluated by the PNMGL, milk production is certainly the most economically important trait.

Records of daily milk production, generally measured at intervals of 30 days, are used for the estimation of 305-day cumulative milk yield (Y305). In Brazil, the latter is applied as a selection criterion in genetic evaluations not only of Gyr dairy cattle, but also of other breeds such as Guzerat dairy, Girolando and Holstein. At present, there is a consensus to use genetic evaluation models that include test-day milk yield (TDMY) instead of Y305, since these models increase the accuracy of selection (Meyer, 2004) and permit the inclusion of incomplete lactations without the need for projections, thus permitting more frequent evaluations and reducing the generation interval (Swalve, 2000).

One alternative to the use of TDMY is a multivariate model that considers production in each of the 10 months of lactation as a single trait. However, in view of the high computational requirements and difficulty in accurately estimating a large number of parameters, fitting a genetic evaluation model that includes all 10 traits may be limited (Meyer, 2007a). This fact has encouraged the application of reduced rank models at the genetic level, for example, principle component analysis (Kirkpatrick and Meyer, 2004). Principal components are uncorrelated variables whose objective is to explain the maximum amount of variation and that are obtained by linear combinations of a set of correlated traits (Kirkpatrick and Meyer, 2004). Thus, components that show little variation can be ignored, and the number of parameters to be estimated is thereby reduced. Another alternative to reduce the number of estimated parameters are factor-analytic models (Meyer, 2007a). These models permit us to identify common factors that are responsible for the association between traits and that are specific, independent and inherent to each trait (Meyer, 2009).

The objective of the present study was to compare standard multivariate, reduced rank and factor-analytic models for the analysis of TDMY from first lactations of Gyr dairy cows to test their application to genetic evaluations of this breed.

\section{MATERIAL AND METHODS}

\section{Data}

The data used in the study were extracted from the Brazilian National Dairy Cattle Archive. The dataset contained 46,161 TDMY records from 7,453 first lactations of purebred 
Gyr dairy and crossbred (Gyr dairy x Holstein) cows, daughters of 598 Gyr dairy sires, ranging in age from 24 to 60 months and belonging to 270 herds. Only test-day records comprising day 5 to day 305 of lactation were considered. The criteria for inclusion of the cows in the study were: first test-day record obtained within 45 days after calving and the number of testday records higher than three. In addition, contemporary groups (herd-year-season of test day) containing fewer than four cows were eliminated. The months in which the test-day records were obtained were divided into four seasons: 1 = rainy season: December to February; 2 = transition rainy-dry season: March to May; 3 = dry season: June to August, and $4=$ transition dry-rainy season: September to November. The cows were divided into five genetic groups: 1 $=100 \%$ Gyr dairy $(\mathrm{DG}) ; 2=87.5 \% \leq \mathrm{DG}<100 \% ; 3=75 \% \leq \mathrm{DG}<87.5 \% ; 4=62.5 \% \leq \mathrm{DG}$ $<75 \%$, and $5=50 \% \leq \mathrm{DG}<62.5 \%$. The TDMY records were divided into 10 monthly classes of lactation, and each class was considered to be a different trait.

\section{Models}

Seven analyses simultaneously considering the 10 traits were performed. First, a standard multivariate model (M10) was used. Next, three reduced rank models for the additive genetic covariance matrix $\left(\Sigma_{\mathrm{A}}\right)$, fitting the first 2 (PC2), 3 (PC3) or 4 (PC4) principal components, were evaluated. Finally, three models considering a 2- (F2), 3- (F3) or 4-factor (F4) structure for $\Sigma_{\mathrm{A}}$ were evaluated.

Additive genetic and residual effects as random effects, as well as the fixed effects of contemporary group, genetic group, age of cow at calving (linear and quadratic effects) and days in milk (linear effect), were included in all models.

\section{Standard multivariate model}

The following linear mixed model was used:

$$
\mathrm{Y}=\mathrm{X} \beta+\mathrm{Zu}+\mathrm{e}
$$

(Equation 1)

where $y$ is the vector of observations; $\beta$ is the vector of fixed effects; $u$ is the vector of additive genetic random effects; $e$ is the vector of residual random effects, and $X$ and $Z$ are incidence matrices corresponding to the observations for $\beta$ and $u$, respectively. It was assumed that $\operatorname{Var}(\mathrm{u})=\Sigma_{\mathrm{A}} \otimes \mathrm{A}$, where $A$ is the numerator relationship matrix, and that $\operatorname{Var}(e)=R$, where $R$ is the matrix of residual covariances. The equations of the mixed models can be written as follows:

$$
\left(\begin{array}{cc}
X^{\prime} R^{-1} \mathbf{X} & \mathbf{X}^{\prime} \mathbf{R}^{-1} Z \\
Z^{\prime} \mathbf{R}^{-1} \mathbf{X} & Z^{\prime} \mathbf{R}^{-1} \mathbf{Z}+\Sigma_{\mathrm{A}}^{-1} \otimes \mathbf{A}^{-1}
\end{array}\right)\left(\begin{array}{l}
\hat{\boldsymbol{\beta}} \\
\hat{\mathbf{u}}
\end{array}\right)=\left(\begin{array}{l}
\mathbf{X}^{\prime} \mathbf{R}^{-1} \mathbf{y} \\
Z^{\prime} \mathbf{R}^{-1} \mathbf{y}
\end{array}\right)
$$

\section{Reduced rank model}

For principal component analysis, model (I) was modified as described by Meyer and Kirkpatrick (2005):

$$
\mathrm{y}=\mathrm{X} \beta+\mathrm{Z}^{\circ} \mathrm{u}^{\circ}+\mathrm{e}
$$


where $\mathrm{Z}^{\circ}=\mathrm{Z}\left(\mathrm{I}_{\mathrm{NA}} \otimes \mathrm{Q}_{\mathrm{m}}\right)$ and $\mathrm{u}^{\circ}=\left(\mathrm{I}_{\mathrm{NA}} \otimes \mathrm{Q}_{\mathrm{m}}^{\prime}\right) \mathrm{u}$, with $\operatorname{Var}\left(\mathrm{u}^{\circ}\right)=\mathrm{A} \otimes \mathrm{I}_{\mathrm{m}}$ and $\mathrm{Q}_{\mathrm{m}}=\mathrm{E}_{\mathrm{m}}\left(\Lambda_{\mathrm{m}}\right)^{1 / 2}$. Here, $N_{\mathrm{A}}$ is the number of animals included in the analysis; $m$ is the number of principal components fitted; $\mathrm{E}$ is the matrix (order $k \mathrm{x} m$ ) of the first $m$ eigenvectors of $\Sigma_{\mathrm{A}}$, where $k$ is the number of traits considered in the analysis, and $\Lambda$ is the diagonal matrix of the eigenvalues of $\Sigma_{A}$. Thus, the equations of the mixed models can be written as follows:

$$
\left(\begin{array}{cc}
X^{\prime} R^{-1} \mathbf{X} & \mathbf{X}^{\prime} \mathbf{R}^{-1} Z^{\circ} \\
Z^{\circ} \mathbf{R}^{-1} \mathbf{X} & Z^{0} \cdot \mathbf{R}^{-1} \mathbf{Z}^{\circ}+\mathbf{A}^{-1} \otimes \mathbf{I}_{\mathrm{m}}
\end{array}\right)\left(\begin{array}{c}
\hat{\boldsymbol{\beta}} \\
\hat{\mathbf{u}}^{0}
\end{array}\right)=\left(\begin{array}{c}
\mathbf{X}^{\prime} \mathbf{R}^{-1} \mathbf{y} \\
\mathbf{Z}^{0} \mathbf{R}^{-1} \mathbf{y}
\end{array}\right)
$$

\section{Factor-analytic model}

For factorial analysis, model (I) was modified as described by Meyer (2009):

$$
\mathrm{y}=\mathrm{X} \beta+\mathrm{Z}^{*} \mathrm{c}+\mathrm{Zs}+\mathrm{e}
$$

where $\mathrm{Z}^{*}=\mathrm{Z}\left(\mathrm{I}_{\mathrm{NA}} \otimes \Gamma_{\mathrm{m}}\right)$, with $\operatorname{Var}(\mathrm{c})=\mathrm{I}_{\mathrm{m}} \otimes \mathrm{A}$ and $\operatorname{Var}(\mathrm{s})=\Psi \otimes \mathrm{A}$. Thus, $\Gamma$ (order $\left.k \mathrm{x} m\right)$ is the factor loading matrix; $\Psi$ is the variance matrix of specific factors $(s)$, a diagonal matrix of order $k$; c is the vector of common factors of length $m N_{A} ; \mathbf{s}$ is the vector of common factors of length $\mathrm{km}$, and $m$ is the number of common factors fitted. The equations of the mixed models can be written as follows:

$$
\left(\begin{array}{ccc}
\mathbf{X}^{\prime} \mathbf{R}^{-1} \mathbf{X} & \mathbf{X}^{\prime} \mathbf{R}^{-1} Z^{*} & \mathbf{X}^{\prime} \mathbf{R}^{-1} Z \\
Z^{*} \cdot R^{-1} \mathbf{X} & Z^{*} \cdot R^{-1} Z^{*}+I_{m} \otimes A^{-1} & Z^{*} R^{-1} Z \\
Z^{\prime} R^{-1} X & Z^{\prime} R^{-1} Z^{*} & Z^{*} R^{-1} Z^{*}+\Psi \otimes A^{-1}
\end{array}\right)\left(\begin{array}{c}
\hat{\beta} \\
\hat{\mathbf{c}} \\
\hat{\mathbf{s}}
\end{array}\right)=\left(\begin{array}{c}
X^{\prime} R^{-1} \mathbf{y} \\
Z^{*} \cdot R^{-1} \mathbf{y} \\
Z^{\prime} R^{-1} \mathbf{y}
\end{array}\right)
$$

Full rank residual covariance matrices (order $10 \times 10$ ) were considered in all models tested. The covariance components were estimated by the restricted maximum likelihood method using the WOMBAT program (Meyer, 2007b).

\section{Model comparison}

The models were compared using the maximum log-likelihood function $(\log \mathrm{L})$ and two information criteria. Akaike's information criterion adjusted for sample size $\left(\mathrm{AIC}_{\mathrm{C}}\right.$ ) was calculated as proposed by Burnham and Anderson (2004):

$$
A I C_{C}=-2 \log L+2 p\left(1+\frac{p+1}{N-p-1}\right)
$$

where $p$ is the number of parameters (variance components) to be estimated, and $N$ is the number of observations considered in the analysis. Bayesian information criterion (BIC) was calculated as:

$$
B I C=-2 \log L+\log [N-r(\mathrm{X})] p,
$$

where $r(\mathrm{X})$ is the rank of the coefficient matrix for fixed effects. 
The accuracy of the estimation of a genetic principal component (PC) was evaluated as the deviation of the ith eigenvector of the analysis fitting $m$ PC (or factors) in relation to the corresponding value obtained with model M10. This deviation was measured as the angle (in degrees) between the corresponding eigenvectors (Kirkpatrick and Meyer, 2004):

$$
\alpha_{i}=\left(\frac{180}{\pi}\right) \arccos \left(\frac{e_{i, m}^{\prime} e_{i, 10}}{\left|e_{i, m}\right| e_{i, 10}}\right),
$$

where $e_{i, m}$ and $e_{i, 10}$ are the ith eigenvectors estimated with the PCm (or Fm) and M10 models, respectively, and $|$.$| is the vector norm.$

In addition, the models were compared based on similarity between the correlation matrices obtained with model PCm (or Fm) and model M10 (Meyer, 2007a):

$$
\Delta r=\sum_{i=1}^{10} \sum_{j=i+1}^{10} \frac{\left(r_{i j, m}-r_{i j, 10}\right)^{2}}{45},
$$

where $r_{i j, m}$ and $r_{i, 10}$ are the correlation estimates between traits $i$ and $j$ obtained with models $\mathrm{PCm}$ (or Fm) and M10, respectively, and $\Delta \mathrm{r}$ is the square root of the average squared deviation of correlations.

\section{RESULTS AND DISCUSSION}

The overall mean TDMY was $10.03 \mathrm{~kg}$ with a standard deviation of $4.58 \mathrm{~kg}$. The highest production was observed in the second month of lactation (Table 1).

Table 1. Number of records (N), mean, standard deviation (SD), average days in milk (aDIM), and number of

\begin{tabular}{|c|c|c|c|c|c|}
\hline Trait (kg) & $\mathrm{N}$ & Mean & SD & aDIM & NCG \\
\hline $\mathrm{CTD}_{1}$ & 3964 & 11.07 & 4.27 & 18 & 653 \\
\hline $\mathrm{CTD}_{2}$ & 5469 & 11.69 & 4.71 & 45 & 865 \\
\hline $\mathrm{CTD}_{3}^{2}$ & 5553 & 11.19 & 4.74 & 75 & 868 \\
\hline $\mathrm{CTD}_{4}^{3}$ & 5445 & 10.70 & 4.73 & 105 & 855 \\
\hline $\mathrm{CTD}_{5}^{4}$ & 5207 & 10.21 & 4.60 & 135 & 815 \\
\hline $\mathrm{CTD}_{6}^{5}$ & 4947 & 9.66 & 4.44 & 165 & 793 \\
\hline $\mathrm{CTD}_{7}^{6}$ & 4654 & 9.21 & 4.23 & 195 & 748 \\
\hline $\mathrm{CTD}_{8}^{7}$ & 4262 & 8.62 & 4.04 & 225 & 709 \\
\hline $\mathrm{CTD}^{8}$ & 3612 & 8.27 & 3.95 & 255 & 609 \\
\hline $\mathrm{CTD}_{10}$ & 3048 & 7.98 & 3.95 & 285 & 513 \\
\hline
\end{tabular}
contemporary groups (NCG) for the test-day milk yield classes $\left(\mathrm{CTD}_{\mathrm{i}}\right)$ analyzed.

The three largest eigenvalues of the additive genetic covariance matrix obtained with model M10 explained 91.14, 6.18 and $1.33 \%$ of the total genetic variation between animals. These results indicate that a large amount of the genetic variation can be explained by few eigenvalues, which are associated with the principal eigenvectors (principal components). This result was expected in view of the high genetic correlations between productions during different months of lactation.

Log L increased with increasing number of parameters in the model, with the highest value being observed for the full rank model, M10 (Table 2). However, the $\mathrm{AIC}_{\mathrm{c}}$ and BIC 
values indicated M10 to be the worst model among the models tested (Table 2). According to these criteria, the model fitting the first two principal components (PC2), which contained 74 parameters, was the best. The two criteria simultaneously evaluate goodness-of-fit and degree of complexity, and the model chosen showed the best association between goodness-of-fit and degree of complexity. Similar results have been reported by Bignardi et al. (2010), who found that only two principal components are sufficient to model the structure of genetic covariances between TDMY records in Holstein cattle.

Table 2. Number of parameters (P) for different models, maximum $\log$-likelihood $(\log \mathrm{L})$, Akaike $(\mathrm{AIC})$ and Bayesian (BIC) information criteria, estimates of the total variation $\left(\Sigma \lambda_{\mathrm{i}}\right)$, and measures of discrepancy $\left(\sqrt{\Delta \mathrm{r}}, \alpha_{\mathrm{i}}\right)$ to estimates from analysis M10.

\begin{tabular}{|c|c|c|c|c|c|c|c|c|c|c|}
\hline \multirow[t]{2}{*}{ Model } & \multirow[t]{2}{*}{$\mathrm{P}$} & \multirow[t]{2}{*}{$\log \mathrm{L}^{\mathrm{a}}$} & \multirow[t]{2}{*}{$\mathrm{AIC}_{\mathrm{c}}^{\mathrm{a}}$} & \multicolumn{5}{|c|}{ Genetic } & \multicolumn{2}{|c|}{ Residual } \\
\hline & & & & $\mathrm{BIC}^{\mathrm{a}}$ & $\Sigma \lambda_{\mathrm{i}}$ & $\sqrt{\Delta \mathrm{r}}$ & $\alpha_{1}$ & $\alpha_{2}$ & $\Sigma \lambda_{\mathrm{i}}$ & $\sqrt{\Delta \mathrm{r}}$ \\
\hline PC2 & 74 & -9.9 & 0.0 & 0.0 & 15.82 & 0.037 & 0.8 & 4.2 & 75.64 & 0.005 \\
\hline PC3 & 82 & -3.8 & 2.0 & 36.2 & 16.00 & 0.029 & 0.8 & 4.3 & 75.46 & 0.005 \\
\hline $\mathrm{PC} 4$ & 89 & -0.4 & 5.6 & 69.7 & 16.15 & 0.018 & 0.2 & 2.4 & 75.30 & 0.003 \\
\hline M10 & 110 & 0.0 & 26.3 & 180.2 & 16.44 & 0 & 0 & 0 & 75.13 & 0 \\
\hline $\mathrm{F} 2$ & 84 & -3.5 & 3.7 & 46.5 & 16.00 & 0.027 & 0.6 & 4.1 & 75.46 & 0.004 \\
\hline F3 & 92 & -0.2 & 8.4 & 85.3 & 15.99 & 0.024 & 0.6 & 4.7 & 75.36 & 0.004 \\
\hline F4 & 99 & -0.0 & 15.2 & 122.2 & 16.15 & 0.018 & 0.2 & 2.4 & 75.30 & 0.003 \\
\hline
\end{tabular}

$\mathrm{PCm}=$ models analysis fitting the leading $m$ principal components; $\mathrm{F}_{\mathrm{m}}=$ factor-analytic model with $m$ factors; M10 $=$ standard multivariate model; ${ }^{a}$ values scaled as deviation from the respective 'best' values; $\sqrt{\Delta r}=$ square root of the average squared deviation of correlations; $\alpha_{i}=$ angle (in degrees) between estimates of the $i^{\text {th }}$ eigenvectors.

Comparison of the $\log \mathrm{L}$ values obtained for models PCm and Fm showed that the improvement in the goodness-of-fit provided by the additional parameters (specific variances) of the Fm models was very small. The number of specific variances that explained less than $0.01 \%$ of the phenotypic variation was 5, 7 and 10 for analyses considering 2, 3 and 4 factors, respectively. The highest percentage of phenotypic variation explained by a specific variance component was $1.43 \%$ for TDMY, using model F2. Since the traits generally show a high genetic correlation, common factors are able to explain almost all of the genetic variation between animals and the addition of specific factors is therefore not necessary, with PCm models being preferred over Fm models.

In general, a small increase in total genetic variance and a corresponding decrease in total residual variance were observed with an increasing number of parameters in the model (Table 2). The angles between the ith eigenvectors were in general very small for all models studied. A slightly larger angle was observed for the second eigenvector (Table 2), which was however associated with an eigenvalue that only explained a small amount of the total variation. Likewise, the similarity between the correlation estimates obtained by PCm (or Fm) analysis and those obtained with model M10 were high for all models tested (Table 2).

An eigenvector corresponds to the weights given to original traits when the principal component is formed (Meyer, 2007a). The estimates of the first two genetic eigenvectors were compared between the PC2 and M10 models (Figure 1). No significant differences were observed between the two analyses, indicating that the direction of the first principal components was estimated correctly. The first eigenvector assigned positive and almost constant weights to all traits, with its associated breeding value corresponding to the average potential of the animal across lactation (Druet et al., 2003). Selection based on the first eigenvector permits us to change production across lactation in the same direction. On the other hand, the second 
eigenvector assigned increasing weights from the beginning $(-0.56)$ to the end of lactation (0.54). Selection based on this eigenvector permits us to change the shape of the lactation curve (persistency of milk yield) of the population (Olori et al., 1999).

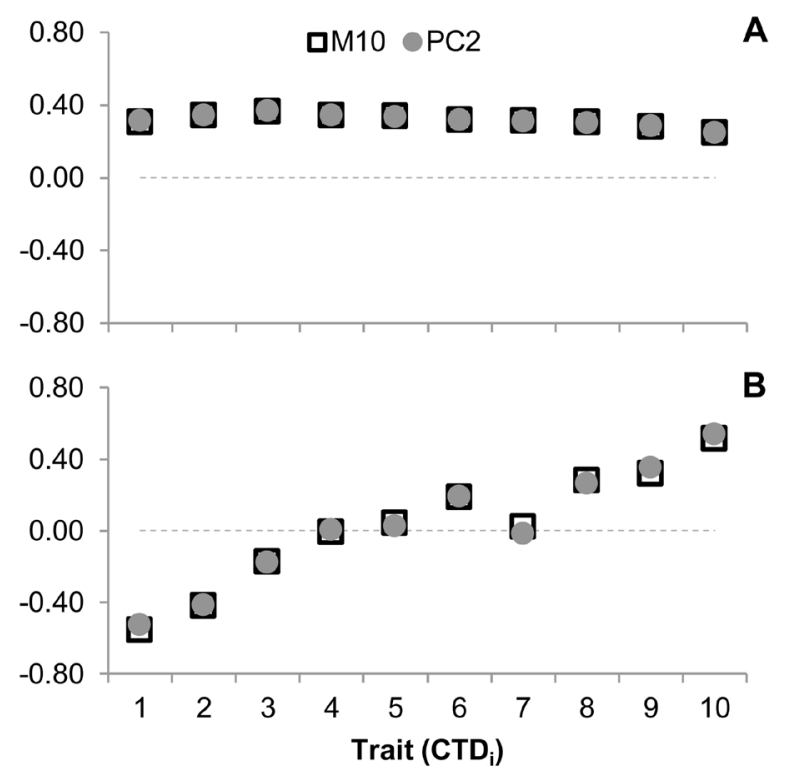

Figure 1. Estimates of the first (A) and second (B) eigenvectors from analysis fitting the first two principal components (PC2) and a full rank, standard multivariate analysis (M10).

The phenotypic variance estimates obtained with models M10 and PC2 were closely similar and ranged from 8.14 to $10.11 \mathrm{~kg}^{2}$. The highest estimates were observed during the first third of lactation. The additive genetic variances showed a similar tendency and ranged from 1.17 to $2.03 \mathrm{~kg}^{2}$. The heritability estimates obtained with models M10 and PC2 are shown in Table 3. Following the tendency of phenotypic and additive genetic variances, heritabilities were higher at the beginning of lactation. Similar estimates have been reported by Pereira et al. (2010), who studied part of the same population using random regression models. Ledic et al. (2002), using TDMY records from the first three lactations of Gyr cattle in two-trait analyses, found heritabilities ranging from 0.14 to 0.24 . Also using two-trait analyses, Herrera et al. (2008) obtained estimates ranging from 0.14 to 0.34 for TDMY of first lactations of Gyr cows. The heritabilities estimated with model PC2 and their respective standard errors were closely similar to those obtained with model M10.

Table 3. Estimates of heritabilities and standard errors from the full rank, standard multivariate analysis (M10), and that fitting the first two principal components (PC2).

\begin{tabular}{lllllllllll}
\hline Model & $\mathrm{CTD}_{1}$ & $\mathrm{CTD}_{2}$ & $\mathrm{CTD}_{3}$ & $\mathrm{CTD}_{4}$ & $\mathrm{CTD}_{5}$ & $\mathrm{CTD}_{6}$ & $\mathrm{CTD}_{7}$ & $\mathrm{CTD}_{8}$ & $\mathrm{CTD}_{9}$ & $\mathrm{CTD}_{10}$ \\
\hline M10 & 0.21 & 0.20 & 0.20 & 0.19 & 0.18 & 0.17 & 0.18 & 0.17 & 0.15 & 0.14 \\
& $(0.04)$ & $(0.03)$ & $(0.03)$ & $(0.03)$ & $(0.03)$ & $(0.03)$ & $(0.03)$ & $(0.03)$ & $(0.03)$ & $(0.03)$ \\
PC2 & 0.21 & 0.20 & 0.20 & 0.18 & 0.18 & 0.16 & 0.16 & 0.16 & 0.15 & 0.13 \\
& $(0.04)$ & $(0.03)$ & $(0.03)$ & $(0.03)$ & $(0.03)$ & $(0.02)$ & $(0.02)$ & $(0.03)$ & $(0.03)$ & $(0.03)$ \\
\hline
\end{tabular}


The genetic correlations between traits (TDMY classes) obtained with the two models were also similar, although slightly higher values were estimated with model PC2 (Figure 2). This finding can be explained by the fact that correlations between traits are forced to have an absolute value of unity when one or few principal components are fitted (Meyer, 2007a). The correlations were close to unity between adjacent classes, decreasing to values of about 0.55 between the first and tenth class. This pattern agrees with those reported by Ledic et al. (2002) and Herrera et al. (2008).
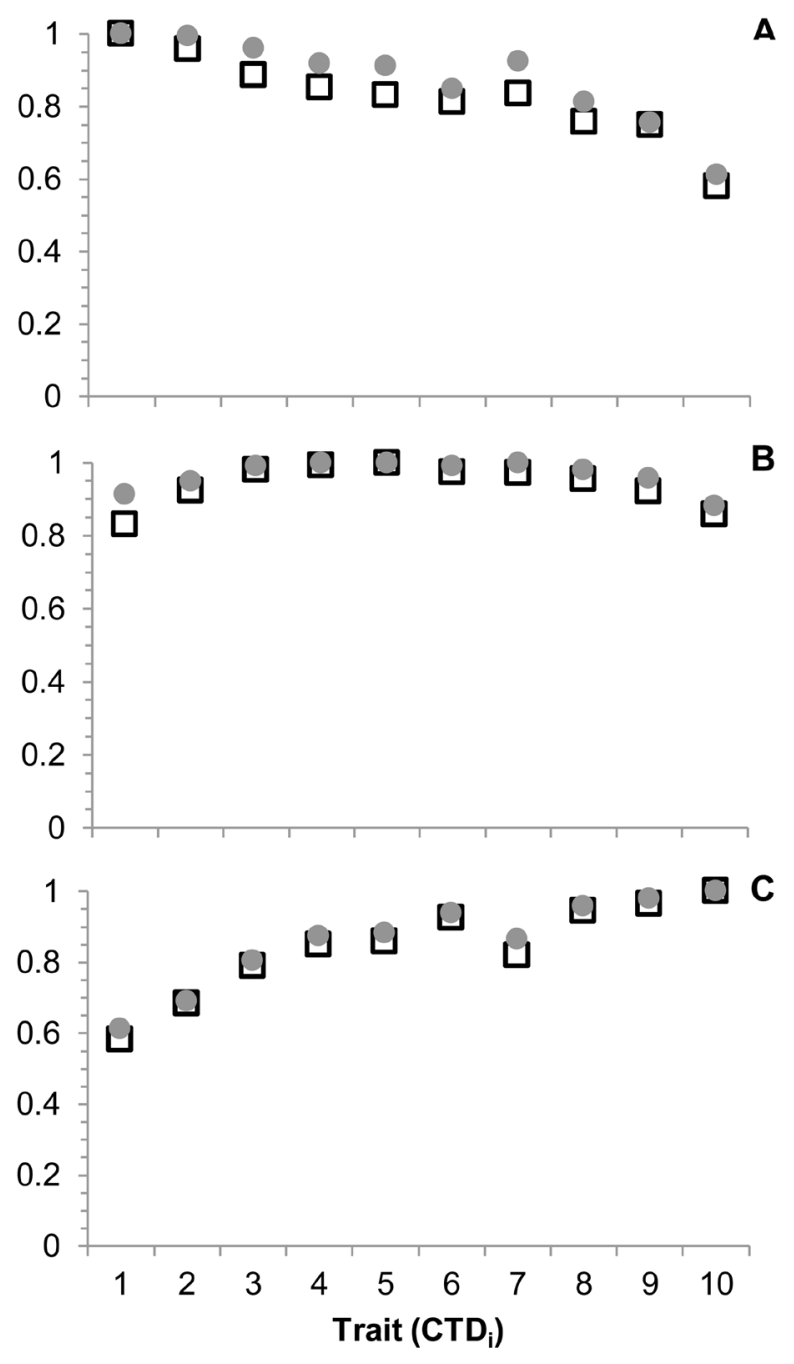

口M10 PC2

Figure 2. Estimates of genetic correlations between the test-day classes $\mathrm{CTD}_{1}$ and $\mathrm{CTD}_{\mathrm{i}}(\mathbf{A}) ; \mathrm{CTD}_{5}$ and $\mathrm{CTD}_{\mathrm{i}}$ (B); $\mathrm{CTD}_{10}$ and $\mathrm{CTD}_{\mathrm{i}}(\mathbf{C})$, from analysis fitting the first two principal components (PC2) and a full rank, standard multivariate analysis (M10). 
The reduction in the number of parameters estimated was significant for model PC2 (74 parameters) when compared to model M10 (110 parameters). One advantage of this reduced number of parameters is the lower computational requirement for the estimation of variance components and genetic evaluation of animals. In addition, model PC2 permitted substantially faster convergence than did model M10.

Taken together, these results show that fitting few principal components is sufficient to model the covariance structure between TDMY records, probably because of the high genetic correlations between most traits (TDMY classes). Although principal component analysis is an interesting option in this case compared to standard multivariate analysis (full rank), the number of parameters to be estimated is still very large. In addition, since genetic evaluations of dairy cattle include the simultaneous analysis of milk, fat and protein yield, as well as somatic cell scores, the large number of parameters and computational requirements for this type of analysis can be a limiting factor. Therefore, further studies using other methods, such as direct estimation of principal components with random regression models, are needed to establish the best approach to model TDMY and to permit its use in genetic evaluations of Gyr dairy cattle.

\section{CONCLUSIONS}

Reduced rank models can be applied to model the structure of covariances between TDMY records. The advantage of these models is the reduced number of parameters to be estimated. The first two principal components are sufficient to explain most of the genetic variation between animals. The use of the factor-analytic model is not indicated in this case since the addition of specific variance components does not significantly improve the goodness-of-fit of the model. Therefore, a model considering the first two principal components is a parsimonious option for the genetic evaluation of Gyr dairy cattle using TDMY under a multivariate framework.

\section{ACKNOWLEDGMENTS}

Research supported by Fundação de Amparo à Pesquisa do Estado de São Paulo (FAPESP), Brazil.

\section{REFERENCES}

Bignardi AB, El Faro L, Rosa GJM, Silva FF, et al. (2010). Multivariate Analyses of First Lactation Test-Day Milk Yield of Holstein Cattle Fitting Factor Analytic and Reduced Rank Models. In $9^{\text {th }}$ World Congress On Genetics Applied To Livestock Production, CDROM, Leipzig.

Burnham KP and Anderson DR (2004). Multimodel inference understanding AIC and BIC in model selection. Sociol. Methods Res. 33: 261-304.

Druet T, Jaffrezic F, Boichard D and Ducrocq V (2003). Modeling lactation curves and estimation of genetic parameters for first lactation test-day records of French Holstein cows. J. Dairy Sci. 86: 2480-2490.

Herrera LGG, El Faro L, Albuquerque LG, Tonhati H, et al. (2008). Parâmetros genéticos para produção de leite no dia do controle e para produção de leite até 305 dias nas primeiras lactações de vacas da raça Gir. Rev. Bras. Zootec. 37 : 1774-1780.

Kirkpatrick M and Meyer K (2004). Direct estimation of genetic principal components: simplified analysis of complex phenotypes. Genetics 168: 2295-2306.

Ledic IL, Tonhati H, Verneque RS, El Faro L, et al. (2002). Estimativa de parâmetros genéticos, fenotípicos e ambientes para as produções de leite no dia do controle e em 305 dias de lactação de vacas da raça Gir. Rev. Bras. Zootec. 31: 
1953-1963.

Meyer K (2004). Scope for a random regression model in genetic evaluation of beef cattle for growth. Livest. Prod. Sci. 86: 69-83.

Meyer K (2007a). Multivariate analyses of carcass traits for Angus cattle fitting reduced rank and factor analytic models. J. Anim. Breed. Genet. 124: 50-64.

Meyer K (2007b). WOMBAT: a tool for mixed model analyses in quantitative genetics by restricted maximum likelihood (REML). J. Zhejiang Univ. Sci. B 8: 815-821.

Meyer K (2009). Factor-analytic models for genotype x environment type problems and structured covariance matrices. Genet. Sel. Evol. 41: 21.

Meyer K and Kirkpatrick M (2005). Restricted maximum likelihood estimation of genetic principal components and smoothed covariance matrices. Genet. Sel. Evol. 37: 1-30.

Olori VE, Hill WG, McGuirk BJ and Brotherstone S (1999). Estimating variance components for test day milk records by restricted maximum likelihood with a random regression animal model. Livest. Prod. Sci. 61: 53-63.

Pereira RJ, Lopes OS, Verneque RS, Santana Jr ML, et al. (2010). Funções de covariância para produção de leite no dia do controle em bovinos Gir leiteiro. Pesq. Agropec. Bras. 45: 1303-1311.

Swalve HH (2000). Theoretical basis and computational methods for different test-day genetic evaluation methods. $J$. Dairy Sci. 83: 1115-1124.

Verneque RS, Panetto JCC, Bruneli FAT, Geraldo CC, et al. (2011). Programa Nacional de Melhoramento do Gir Leiteiro - Sumário Brasileiro de Touros - Resultado do Teste de Progênie - Maio 2011 (Documentos, 145). Embrapa Gado de Leite, Juiz de Fora. 